\title{
Detection of Fatty Acids Profile in Human Saliva with Special Reference to Ovulation
}

\author{
S. Alagendran \\ Department of Animal Science, Bharathidasan University \\ Tiruchirapalli- 24.Tamil nadu, India \\ $\&$ \\ Sensorial physiology laboratory, Department of Physiology \\ Faculty of Medicine, AV.Universidad 3000, C.P, 04510, UNAM, Mexico \\ G. Archunan (Corresponding Author), K. Ramesh kumar \& R. L. Rengarajan \\ Department of Animal Science, Bharathidasan University \\ Tiruchirapalli- 24. Tamil nadu, India \\ Tel: 91-431-240-7040_E-mail: archunan@bdu.ac.in \\ G. Fernandez \\ Department of Pharmacology, Faculty of Medicine \\ AV.Universidad 3000, C.P, 04510, UNAM, Mexico \\ R. G. Guzman \\ Sensorial physiology laboratory, Department of Physiology, Faculty of Medicine \\ AV.Universidad 3000, C.P, 04510, UNAM, Mexico
}

\begin{abstract}
Human saliva was analyzed by Gas-Chromatography (GC) for fatty acids study throughout menstrual cycle. The fatty acid profiles from preovulatory, ovulatory and postovulatory phase's sample were compared to establish any qualitative and quantitative differences that might have potential value in detection of ovulation in women. In the present study, 17 different types of fatty acids were identified. The fatty acids resembling as Oleic acid, Palmitic acid and acetic acid were comparatively higher in concentration than the other fatty acids. These three fatty acid were mostly present in ovulatory phase ${ }_{2}$ to be higher when compared to follicular and luteal phases of menstrual cycle bear out the cyclic fluctuations in lipid levels do occur under the influence of both endogenous and exogenous sex hormones. The results imply that the variation in the fatty acids is unswervingly/ obliquely correlated to the phase of menstrual cycle.
\end{abstract}

Keywords: Fatty acids, Gas chromatography, Women, Saliva, Ovulation

\section{Introduction}

The detection of ovulation simply and precisely is essential for natural family planning (NFP) methods and is useful in cases of infertility. Most cycles in fertile women show a typical pattern of increasing preovulatory estrogen levels that is associated with a luteinising hormone (LH) surge and a subsequent progesterone rise. However, we have observed individual differences in this typical pattern, (Alliende, 2002) which sometimes may make accurate ovulation detection difficult. Most NFP methods consider these personal variants. perspective of establishing any potential role of the normal variation in estrogen and progesterone on substrate metabolism as well as establishing the necessity to control for sex steroid hormone status in metabolic studies including women. In general, the majority of studies have shown that the normal cyclic fluctuations in estrogen and progesterone observed throughout the menstrual cycle do not affect whole body lipid or carbohydrate oxidation, at rest or during moderate exercise nevertheless, menstrual cycle phase may affect substrate metabolism under certain 
conditions. It is possible that varying rates of fatty acid oxidation may provide a further means by which specific fatty acids exert different overall metabolic effects (Pschera et al, 1989).

In different studies of many cycles in healthy volunteers, urine LH, although highly concordant with serum LH, does not always show a clear LH surge, because of multiple or ill-defined surges (Baird, 1991). We have had the same experience (Alliende, 2002), and a Indian multicenter study has confirmed this finding with ultrasound studies in a large population (Ecochard et al, (2001) and Baird et al, (1991) have established, in their fertile window studies, that a day of luteal transfer, after a rapid drop in the ratio of estrogen and progesterone biological fluid metabolites like blood and urine, is a better ovulation indicator than the LH surge. Up to date, the importance has been paid on the biochemical importance of saliva in the view of noninvasive aid for detecting the diagnostic aid for women fertility. Hormones used to measure in blood have now been estimated in saliva, though the quantities are comparatively less (Braat et al, 1991, Tabak, 2001). Hence, saliva is considered as the best non-invasive source for chemical and biochemical study including fatty acid analysis (Freundl et al, 1996). Reports show that saliva is a very good source of both hormones and fatty acid and their levels changed in accordance with the menstrual cycle (Flynn and Lynch, 1974).

Saliva has become popular in recent decades as a medium for the measurement of numerous biomolecules (Streckfus and Bigler, 2002). The greatest advantage, when compared to blood sample collection, is that saliva is readily accessible and easy to use for each biochemical investigation. Consequently, it can be used in clinically difficult situations, such as in children, handicapped, and anxious patients, where blood sampling could be a difficult act to perform (Aps and Martens, 2005). A wide range of biomolecules are now monitored in saliva (Lawrence, 2002 and Tabak, 2001). Lipids and fatty acids were extensively studied in saliva for biomarker research; these include the quantification of steroid hormones such as cortisol or progesterone (Vining and McGinley, 1987; Ellison, 1993; Aardal and Holm, 1995; Castro et al, 2000), and the detection of various therapeutic or illicit drugs (Kintz et al, 2000). Reliable and accurate measures of fatty acids and its volatiles are easily detected through gas chromatography.

It is well known that the lipids and their derivatives are involved in the olfactory communication. For instance, free fatty acids (FFA) seem to function as pheromones in a number of mammals including tiger (Bramhachary et al., 1991, 1992) and African Cheetah (Poddar-Sarkar and Bramhachary, 1997). In addition, the free fatty acids reported in the tiger urine can serve as the basis for individual distinction. Mattina et al., (1991) noticed that bobcat urine contains palmitic and octanoic acids that are involved in several behaviours. The substance present in the vaginal secretions of estrogen-stimulated rhesus monkey 'couplins' contains short chain aliphatic acids such as acetic, prop ionic, isobutyic, n-butyric, and isoalanine seems to evoke mounting and ejaculatory behavior (Michael, 1975; Diani et al, 1998). Volatile fatty acids such as, acetic, proponic, butanoic acids are identified in normal physiologic constituents of the vaginal secretions in healthy young women with regular menstrual cycles. These acids vary during menstrual cycle and reaching a peak near the time of ovulation, as they do in infra-primates (Preti, et al, 2003). The aim of this work is to evaluate the different fatty acid components of human saliva obtained during ovulation and at the term of pregnancy

It is of interest that similar changes may occur in salivary fatty acids constituents of women during various reproductive stages, which may act as sex arousal for males of the same species (Martin et al, 2005). Detection of ovulation is the major problems in artificial insemination treat in women. The reason behind this study was to identify the ovulatory specific fatty acid(s) in women and make it possible to develop a biochemical marker to detect ovulatory phase accurately. Therefore, in the present study, the fatty acid profiles are compared between ovulatory phase saliva with that of follicular and luteal phase saliva in order to elucidate the ovulatory specific fatty acids.

\section{Materials and methods}

\subsection{Samples}

We obtained samples from 60 healthy women of reproductive age $(20$ - 40 years) who were not taking oral contraceptives or any other medication. For comparisons of whole saliva with the ultra filtrate, we used the collector immediately after the collection of whole saliva (Navazesh, 1993). The subjects were asked to accumulate saliva in their mouths over 8 to $10 \mathrm{~mm}$ without swallowing and then to expel the saliva into a polypropylene container. No stimulants were used in this procedure. All samples were stored at $-20{ }^{\circ} \mathrm{C}$ until assayed. Whole saliva were centrifuged (1000 x g for $10 \mathrm{mins}$ ) before assay to remove particulate matter.

One individual collected saliva with the collector over a period of two menstrual cycles at home between 0800 and 900 hours. The samples were stored in the home freezer immediately after collection and brought to the laboratory once every two weeks. The anthropometric and biochemical variable index was also analyzed in each 
phases of reproductive period (Age, weight, BMI, systolic and diastolic pressure, cholesterol, triglycerides, phospholipids and free fatty acid profile followed by GC.

\subsection{Biochemical analysis}

Total cholesterol was estimated by the method of Zlatkis (1935), Triglycerides were estimated by the method of Foster and Dunn (1973) and Phospholipids were estimated by the method of (Rouser et al., 1970). Every biochemical variables were assayed using UV spectrophotometer (Perkin Elmer, LAMBDA 1050 spectrophotometer - USA) at the laboratory of Centre for pheromone technology, Department of Animal science, Bharathidasan University, Tiruchirappalli, Tamil nadu, India in triplicate assay of all reproductive phases.

Sample preparation for salivary fatty acids using Gas Chromatography analysis

The lipid was extracted from the collected samples by using chloroform and methanol (1:1) and the total lipid was estimated by Folch et al., (1957). The lipids were subjected for fatty acid analysis using Gas Chromatography. $1 \mathrm{ml}$ of saponification reagent was added in a teflon lined screw cap tubes along with the lipid extract. The tubes were tightly closed and kept for $30 \mathrm{mins}$ at $100^{\circ} \mathrm{C}$ in a water bath. $2 \mathrm{ml}$ of methylation reagent was added to each tube and kept again in a water bath at $80^{\circ} \mathrm{C}$ for 20 mins. The tubes were closed down to the ambient temperature. Extracting solvent $(1.25 \mathrm{ml})$ was added to each tube, closed tightly and shaken thoroughly for 10 mins. About 2/3 of the organic phase (upper layer) containing the fatty acid methyl esters were transferred into screw cap glass vials. From each vial $1 \mu \mathrm{l}$ of the fatty acid methyl ester (FAME) was injected into the gas chromatography (GC) column (DEGS) using column temperature of $180^{\circ} \mathrm{C}$ and injection port temperature was $200^{\circ} \mathrm{C}$. Flame Ionization Detector (FID) was used as a detector with $230^{\circ} \mathrm{C}$. Nitrogen was used as reagent gas (Miller and Berger, 1985).

\subsection{Statistical analysis}

All experiments were determined by one way ANOVA followed by Tukey test comparison procedure. Data were expressed as means and standard error $(\mathrm{x} \pm \mathrm{SE})$. Differences with $\mathrm{p}<0.05$ and $\mathrm{p}<0.01$ were considered statistically significant (Microsoft STATISTICA ver. 5.0, Stat. Soft. Inc. 1995).

\section{Results and Discussion}

The total cholesterol content indicates as $87.65 \pm 4.73 \mathrm{mg} / \mathrm{ml}(\mathrm{F}=2.345, \mathrm{p} \leq 0.05)$ highly significant in ovulatory phase compare to follicular and luteal phases, and phospholipids were lower during the luteal phase as compared with the follicular phase (Not significant at $\mathrm{p} \leq 0.05)$, and Triglycerides levels were highest around ovulation $(\mathrm{F}=$ 1.786; $\mathrm{P}<0.05$ ). More women were classified above the desirable range (Triglycerides $>/=73 \mathrm{mg} / \mathrm{ml}$ or total cholesterol $>/=200 \mathrm{mg} / \mathrm{ml}$ ) when measured during the follicular phase according to the range of serum levels comparatively less. The anthrophotometric index shows age, weight in grams, basal mass index of women and blood pressure range are all vary from follicular phase to all other phases (Fig.1). For instance, blood pressure intensity was highly significant during ovulatory phase as compare to other phases $(p \leq 0.01)$ (Table. 1$)$. The analysis of human saliva using GC shows 16 different fatty acids. Some of the free fatty acids such as Oleic and, Acetic acid, Linoleic acid, myristic acid, lauric acid, palmitic acid and butyric acid were present in all the three stages. Among these oleic acid, acetic, palmitic, buytric and lauric acids were found to be higher concentration in ovulatory phase and luteal phase when compared to other fatty acids (Fig.2). The two fatty acids, arachidonic acid and eicospentaenoic acid were higher in amount in all the three phases although these fatty acids were comparatively more in ovulatory phase. And also stearic, myristic, butyric, caproic and linoleic acids were found in luteal and follicular phase while exclusively absent in the ovulatory period (Table 2).

In the present investigation the variation in lipid content across various reproductive phases clearly indicates that lipids have got specific role in olfactory communication depending upon the physiological state of the individuals and responders. The mechanism of fixing volatile molecules by "lipids" has been observed in Tulpaia belangeri (Shrew), one of the most primitive mammals (Stralendorff, 1987, Miyazawa, et al, 2009). In certain cases lipids itself act as pheromones. For instance, our previous study also indicated that (Kannan and Archunan, 1998) lipids play a major role in the sexual attraction in Rattus norvigicus.

The present study revealed that the free fatty acids such as Linoleic acid, myristic acid, lauric acid, palmitic acid and butyric acid were present in all the three phases of reproductive cycle in women. Among these, oleic acid, acetic, palmitic, buytric and lauric acids are predominantly present in higher concentration in estrus phase. It indicates that the appearance of these fatty acids in ovulatory phase and luteal phases are due to the high circulation of steroidal hormones. The difference in the fatty acid composition may be due to the alterations of lipid metabolism especially of metabolic events affecting ketogenesis and would have reflected in the chemical alteration of urine. The presence of fatty acids like lauric and linolenic with uniform concentration throughout 
the menstrual cycle showed that these fatty acids are not influenced by the hormonal changes during menstrual cycle.

The notable amount of palmitate during ovulatory phase in comparison to that of other reproductive phases suggests that it may be involved in chemo attraction. Since the ovulatory phase of women saliva has the capacity to attract the opposite partner through pheromonal signals, presence of these fatty acids in ovualtory phase appears to play a role in the day 13. This finding is consistent with the report of Mattina et al., (1991) that palmitic acid was excreted in bobcat urine and is involved in sexual attraction of conspecifics. Brahmachary et al., (1992) also stated that the free fatty acids seem to function as pheromones in tigers. It is also to be remembered that the heptanoic and isohexanoic fatty acids, which are present in tiger (Bramhachary et al., 1991, 1992) and Cheetah (Poddar-Sarkar and Bramhachary, 1997) as marking fluid but these fatty are absent in human saliva.

Furthermore, the variation in fatty acid concentration of urinary samples may be serving as the basis for individual recognition. The present suggestion may recall with earlier findings of Welsch et al., (1988); Wise et al., (2007); Decker et al., (1992) and (Preti et al., (2003) in which the sebaceous glands of rabbit, rat, and fishing bat have many kinds of fatty acids, esters and glycerides which are appeared to be involved in individual recognition and scent marking. In the present study, the saturated fatty acids like lauric, tridecanoic acids are predominantly found in almost all the urine samples. By contrast, the bobcat urine predominantly contains unsaturated fatty acids (Mattina et al., 1991). Likewise, available report indicates that the mice glandular tissue like preputial and cheek contain long chain double bonded fatty alcohols (Albone, 1984).

Even though the present study did not expose any estrus (ovulatory) specific salivary fatty acids, it is interesting to note that the concentration of certain fatty acids seems to be higher during ovulatory phase. This finding indicates that the quantification of salivary fatty acids may be considered as one of the parameters in detection of ovulation.

\section{Acknowledgement}

This work was partially supported by the grant from DBT and UGC-SAP, Government of India.

\section{References}

Albone, E.S. (1984). The investigation of chemical signals between mammals. In: Mammalian Semio-chemistry. Wiley-Interscience Chiehester U.K. pp 75-76.

Alliende ME. (2002). Mean versus individual hormonal profiles in the menstrual cycle. Fertil Steril, 78(1): 90-95.

Aps JK, Martens LC. (2005). The physiology of saliva and transfer of drugs into saliva. Forensic Sci Int., 10; 150(2-3): 119-131.

Baird DT. (1991). Abortion rates still rising. BMJ. 7; 303(6802): 579.

Braat DD, Smeenk JM, Manger AP, Thomas CM, Veersema S, Merkus JM 1. (1998). Saliva test as ovulation predictor. Lancet, 352: 1283-1284.

Brahmachary, R.L. Dutta. J. and Poddar-Sarkar, M. (1991). The marking fluid of tiger. Mammalia, 55: 150.

Brahmachary, R.L., Poddar-Sarkar, M. and Dutta, J. (1992). Chemical signal in the tiger. In: Chemical signals in vertebrate VI. D. Muller-Schware and R.L. Doty (Eds), Plenum press. New York. pp 477-479.

Decker, D.M., ringelberg, D. And White, D.C. (1992). Lipid components in anl scent sacs of three mongoose species (Helogale parvula, Crossarchus obscurus, Suricatta suricatta). J.Chem. Ecol., 18: 1511-1524.

Diani F, Cacco M, Molinaroli A, Cerruti G, Meloncelli C, Turinetto A. (1998). Fatty acid composition of the cervical mucus obtained during ovulation and at the term of pregnancy. Minerva Ginecol, 50 (10): 405-410.

Ecochard R, Boehringer H, Rabilloud M, Marret H. (2001). Chronological aspects of ultrasonic, hormonal, and other indirect indices of ovulation. BJOG. 108(8): 822-829.

Foster L.B, and Dunn R.T. (1973). Stable reagents for determination of serum triglycerides by a colorimetric Hantzsch condensation method. Clin Chem., 19: 38-40.

Freundl G, Bremme M, Frank-Herrmann P, Baur S, Godehardt E, Sottong U. (1996). The CUE Fertility Monitor compared to ultrasound and LH peak measurements for fertile time ovulation detection. Adv Contracept, 12: $111-121$ 
Flynn AM, Lynch SS. (1976). Cervical mucus and identification of the fertile phase of the menstrual cycle. $B r J$ Obstet Gynaecol, 83: 656-659.

Folch, J., Less, M. and Slone-Stanley, G.H. (1957). A simple method for the isolation and purification of total lipid from animal tissues. J. Biol. Chem, 226: 497-498.

Illingworth PJ, Reddi K, Smith KB, Baird DT. (1991). The source of inhibin secretion during the human menstrual cycle. J Clin Endocrinol Metab, 73(3): 667-673.

Kannan, S. and Archunan, G. (1998). Effect of gonadectomy on scent glands pheromonal orientation and grooming behaviour in rats, Rattus norvegicus. J. Exp. Zool. India, 1: 149-158.

Lawrence HP. (2002). Salivary markers of systemic disease: noninvasive diagnosis of disease and monitoring of general health. J Can Dent Assoc., 68(3): 170-174.

Martins Y, Preti G, Crabtree CR, Runyan T, Vainius AA, Wysocki CJ. (2005). Preference for human body odors is influenced by gender and sexual orientation. Psychol Sci., 16(9): 694-701.

Mattina, M.J.I., Pignatello, J.J. and Swihart, R.K. (1991). Identification of volatile components of Bobcat (Lynx rufus) urine. J. Chem. Ecol., 17 (2): 451-462.

Michael, R.P. (1975). Hormonal steroids and sexual communication in primates. J. Steroid Biochem., 6: 161-170.

Miller, L. and Berger, T. (1985). Bacteria identification by GC of whole cell fatty acids, GC Hewlett packard Appl. Note, 228-241.

Miyazawa T, Gallagher M, Preti G, Wise PM. (2009). Odor detection of mixtures of homologous carboxylic acids and coffee aroma compounds by humans. J Agric Food Chem., 1; 57(21): 9895-9901.

Navazesh M. (1993). Methods for collecting saliva. Ann N Y Acad Sci., 20: 72-77.

Poddar-Sarkar M \& Brahmachary R.L. (1997). J Lipid Med Cell Sign, 15-285.

Preti G, Wysocki CJ, Barnhart KT, Sondheimer SJ, Leyden JJ. (2003). Male axillary extracts contain pheromones that affect pulsatile secretion of luteinizing hormone and mood in women recipients. Biol Reprod, 68(6):2107-2113.

Pschera H, Larsson B, Kjaeldgaard A. (1989). Changes in fatty acid composition of cervical mucus lecithin during pregnancy. Gynecol Obstet Invest., 28(3): 118-122.

Rouser, G., Fkeischer S, and Yamamoto, A. (1970). Two dimensional thin layer chromatographic separation of polar lipids and determination of phospholipids by phosphorus analysis of spots. Lipids, 5: 494-496.

Streckfus C.F, and Bigler L.R. (2002). Saliva as a diagnostic fluid. Oral Dis., 8: 69-76.

Stern K, and McClintock MK. (1998). Regulation of ovulation by human pheromones. Nature, 12: 177-179.

Stralendroff, F.V. (1987). Partial chemical characteristic of urinary signalling pheromone in tree shrew, Tupaia belangeri. J.Chem. Ecol., 13: 655-697.

Tabak LA. (2001). A revolution in biomedical assessment: the development of salivary diagnostics. $J$ Dent Educ., 65(12): 1335-1339.

Welsh, C.J., Moore, R.E., Barlet, R.J and Jackson, L.L. (1988). Novel species typical estersfrom preputial glands of sympatric voles, Micxrotus monotanus and M. Pennsylvanicus. J. Chem. Ecol., 14: 143-158.

Wise PM, Miyazawa T, Gallagher M, Preti G. (2007). Human odor detection of homologous carboxylic acids and their binary mixtures. Chem Senses, 32(5):475-482.

Zlatkis A, Zak B, Boyle A.J. (1953). A new method for the direct determination of serum cholesterol. J Lab Clin Med., 41: 486-492. 
Table 1. Indicates the anthropometric and biochemical variables during menstrual cycle

\begin{tabular}{|l|l|l|l|l|}
\hline $\mathrm{N}=60$ subjects & \multicolumn{1}{|c|}{$\begin{array}{c}\text { Follicular phase } \\
(6-12 \text { days })\end{array}$} & \multicolumn{1}{|c|}{$\begin{array}{c}\text { Ovulatory phase } \\
(13-14 \text { days })\end{array}$} & $\begin{array}{c}\text { Luteal phase }(15-26 \\
\text { days })\end{array}$ & P value \\
\hline Age & $23.4 \pm 1.23$ & $25.78 \pm 2.23$ & $21.22 \pm 1.87$ & $\mathrm{P} \leq 0.01$ \\
\hline Weight $(\mathrm{kg})$ & $45.4 \pm 1.67$ & $67.8 \pm 4.78$ & $55.76 \pm 2.14$ & $\mathrm{P} \leq 0.01$ \\
\hline BMI $(\mathrm{kg} / \mathrm{m} 2)$ & $24.7 \pm 1.89$ & $43.76 \pm 2.34$ & $30.65 \pm 2.13$ & $\mathrm{P} \leq 0.01$ \\
\hline $\begin{array}{l}\text { Systolic blood } \\
\text { pressure (mmHg) }\end{array}$ & $115.7 \pm 9.65$ & $120.45 \pm 12.7$ & $90.87 \pm 7.65$ & $\mathrm{P} \leq 0.01$ \\
\hline $\begin{array}{l}\text { Diastolic blood } \\
\text { pressure (mmHg) }\end{array}$ & $56.7 \pm 3.45$ & $76.89 \pm 6.54$ & $61.76 \pm 4.78$ & $\mathrm{P} \leq 0.01$ \\
\hline Cholesterol $(\mathrm{mg} / \mathrm{ml})$ & $67.6 \pm 2.34$ & $87.65 \pm 4.73$ & $78.6 \pm 2.98$ & $\mathrm{P} \leq 0.05$ \\
\hline Triglyceride (mg/ml) & $54.78 \pm 1.98$ & $73.56 \pm 3.76$ & $60.87 \pm 2.42$ & $\mathrm{P} \leq 0.05$ \\
\hline Phospholipids & $43.34 \pm 2.24$ & $56.73 \pm 2.78$ & $39.87 \pm 1.98$ & $\mathrm{NS}$ \\
\hline
\end{tabular}

Data are Mean \pm S.E in triplicate analysis of all reproductive phases by evaluate through one way ANOVA-

Tukey test shows significantly different from follicular towards ovulatory phase value.

Table 2. Fatty acid profile in human saliva during menstrual cycle

\begin{tabular}{|l|c|c|c|}
\hline \multicolumn{1}{|c|}{ Fatty acid (mg/ml) } & $\begin{array}{c}\text { Follicular phase (6-12 } \\
\text { days) }\end{array}$ & $\begin{array}{c}\text { Ovulatory phase (13-14 } \\
\text { days) }\end{array}$ & $\begin{array}{c}\text { Luteal phase (15-26 } \\
\text { days) }\end{array}$ \\
\hline C2 - Acetic acid & $7.32 \pm 1.22$ & $13.43 \pm 3.67$ & $10.9 \pm 2.23$ \\
\hline C4 - Butyric acid & $9.32 \pm 1.12$ & $8.13 \pm 0.87$ & $6.76 \pm 1.54$ \\
\hline C6 - Caproic acid & $0.25 \pm 0.01$ & $0.13 \pm 0.07$ & $4.82 \pm 0.12$ \\
\hline C12 Lauric acid & $2.23 \pm 1.09$ & $1.98 \pm 0.98$ & $2.81 \pm 1.12$ \\
\hline C14 Myristic acid & $6.78 \pm 2.24$ & $3.65 \pm 1.06$ & $2.55 \pm 0.013$ \\
\hline C14:1 Myristoleic acid & $1.35 \pm 0.87$ & $1.22 \pm 0.45$ & $1.31 \pm 0.65$ \\
\hline C16 Palmitic acid & $7.56 \pm 2.78$ & $13.32 \pm 4.77$ & $9.34 \pm 2.87$ \\
\hline C16:1 Palmitoleic acid & $1.00 \pm 0.65$ & $0.34 \pm 0.06$ & $0.43 \pm 0.10$ \\
\hline C18 Stearic acid & $3.22 \pm 2.08$ & $0.56 \pm 0.07$ & $4.34 \pm 1.41$ \\
\hline C18:1 Oleic acid & $6.56 \pm 2.67$ & $24.56 \pm 5.13$ & $8.50 \pm 3.78$ \\
\hline C18:2 Linoleic acid & $0.36 \pm 0.12$ & $0.31 \pm 0.08$ & $4.45 \pm 1.26$ \\
\hline C18:3 Linolenic acid & $0.78 \pm 0.16$ & $0.65 \pm 0.21$ & $0.37 \pm 0.17$ \\
\hline C20 Arachidic acid & $0.87 \pm 0.23$ & $0.34 \pm 0.12$ & $4.80 \pm 2.12$ \\
\hline C20:4 Arachidonic acid & $4.89 \pm 0.33$ & $4.73 \pm 0.44$ & $4.96 \pm 0.18$ \\
\hline C20.5 Eicosapentaenoic & $3.99 \pm 0.34$ & $3.78 \pm 0.16$ & $3.89 \pm 0.19$ \\
\hline acid. & & & $2.54 \pm 0.97$ \\
\hline C22 Behenic acid & $0.98 \pm 0.18$ & $0.89 \pm 0.15$ & $0.98 \pm 0.21$ \\
\hline C24 Lignoceric acid & $0.87 \pm 0.56$ & $0.78 \pm 0.34$ & \\
\hline
\end{tabular}




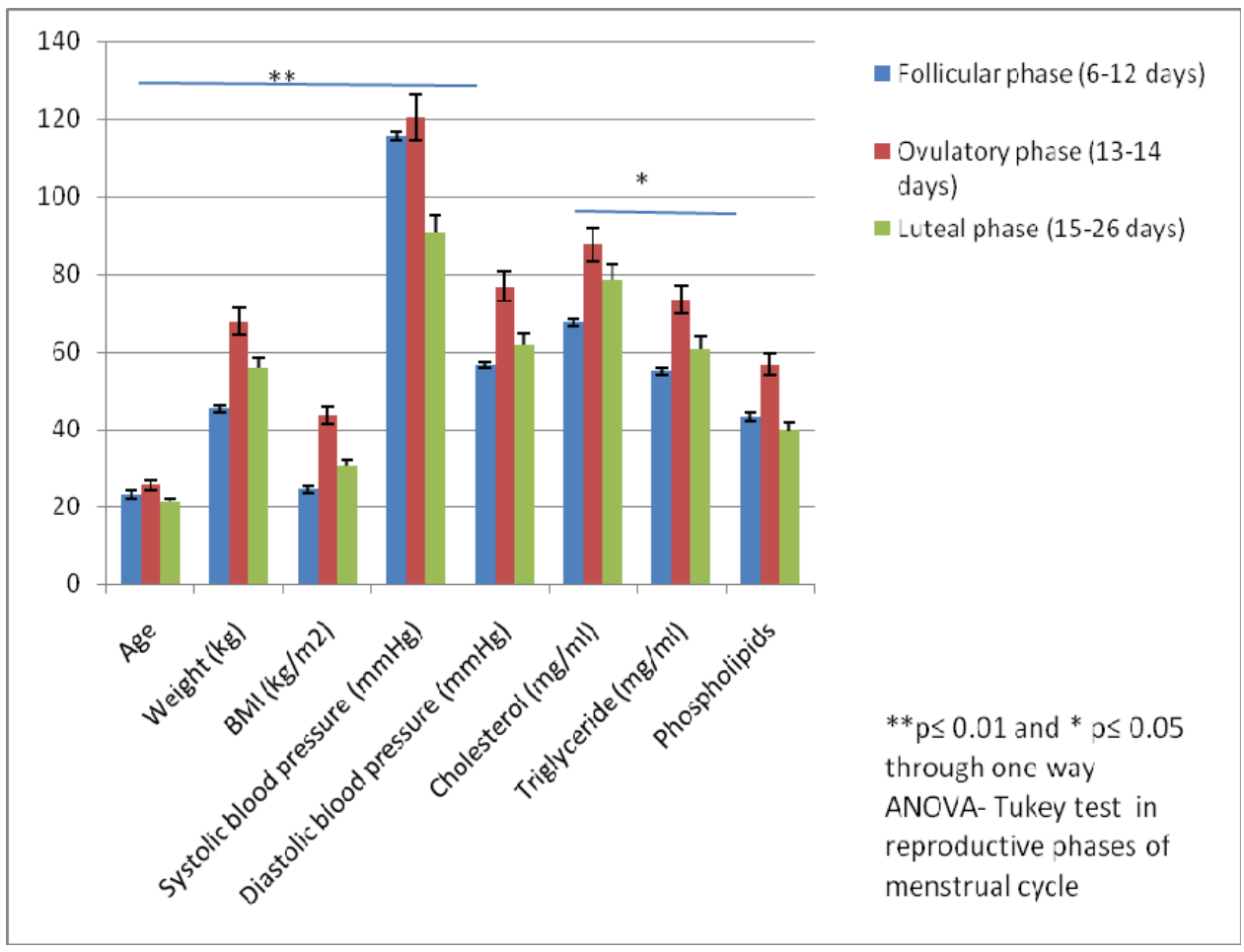

Figure 1. Anthropometric and Biochemical variable graph during menstrual cycle $(\mathrm{N}=60)$

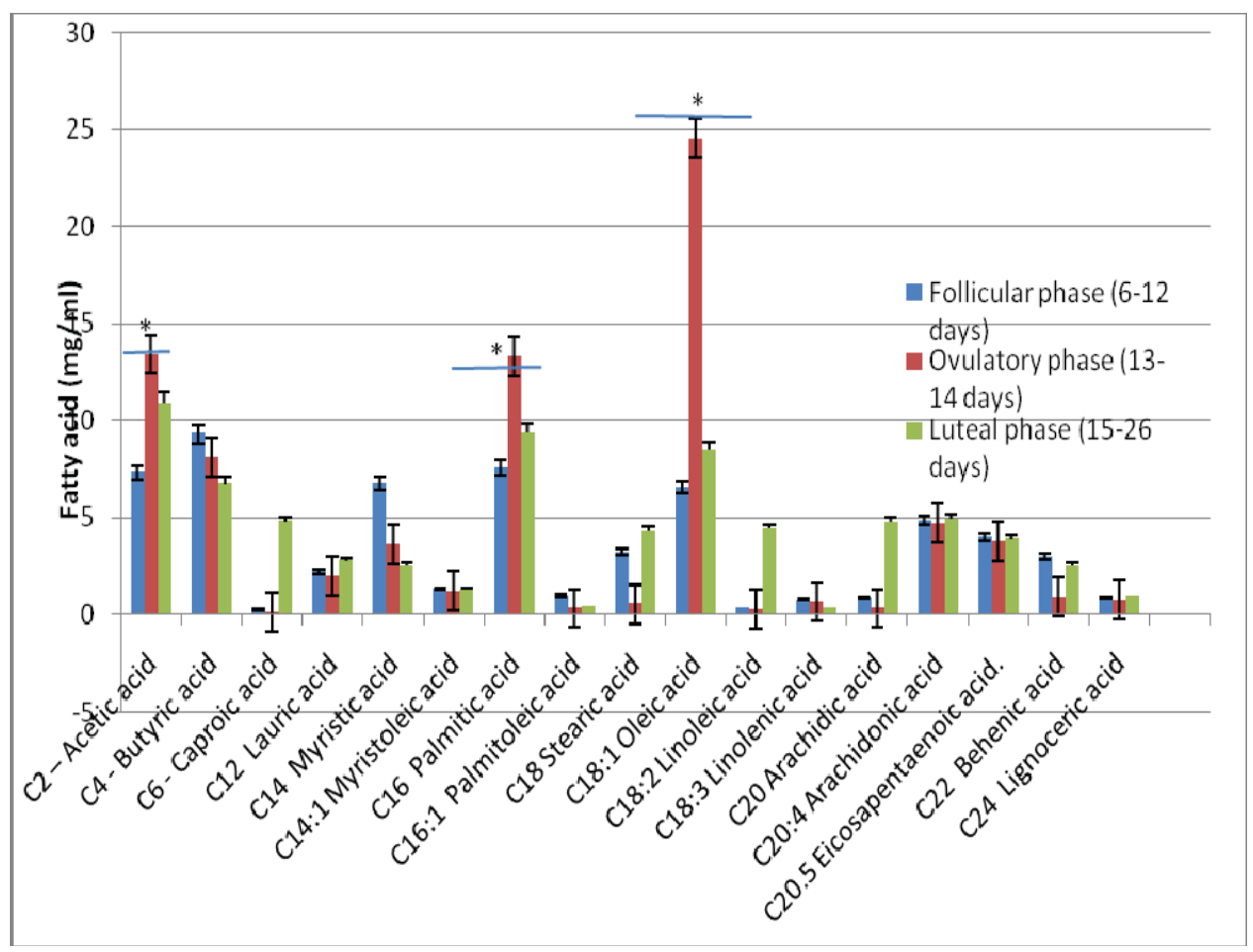

Figure 2. Fatty acid profile in saliva during reproductive phases of menstrual cycle

${ }^{*} \mathrm{p} \leq 0.05$ significantly higher in ovulatory phase as compared to follicular and luteal phases. 